\title{
Neuroanatomical Markers of Social Hierarchy Recognition in Humans: A Combined ERP/MRI Study
}

\author{
Hernando Santamaría-García, ${ }^{\star}$ Miguel Burgaleta, ${ }^{\star}$ and Nuria Sebastián-Gallés \\ Center for Brain and Cognition, Department of Technology, Universitat Pompeu Fabra, 08018, Barcelona, Spain
}

\begin{abstract}
Social hierarchy is an ubiquitous principle of social organization across animal species. Although some progress has been made in our understanding of how humans infer hierarchical identity, the neuroanatomical basis for perceiving key social dimensions of others remains unexplored. Here, we combined event-related potentials and structural MRI to reveal the neuroanatomical substrates of early status recognition. We designed a covertly simulated hierarchical setting in which participants performed a task either with a superior or with an inferior player. Participants showed higher amplitude in the N170 component when presented with a picture of a superior player compared with an inferior player. Crucially, the magnitude of this effect correlated with brain morphology of the posterior cingulate cortex, superior temporal gyrus, insula, fusiform gyrus, and caudate nucleus. We conclude that early recognition of social hierarchies relies on the structural properties of a network involved in the automatic recognition of social identity.
\end{abstract}

Key words: brain morphology; EEG; event-related potentials; MRI; social hierarchy; status

\section{Significance Statement}

Humans can perceive social hierarchies very rapidly, an ability that is key for social interactions. However, some individuals are more sensitive to hierarchical information than others. Currently, it is unknown how brain structure supports such fast-paced processes of social hierarchy perception and their individual differences. Here, we addressed this issue for the first time by combining the high temporal resolution of event-related potentials (ERPs) and the high spatial resolution of structural MRI. This methodological approach allowed us to unveil a novel association between ERP neuromarkers of social hierarchy perception and the morphology of several cortical and subcortical brain regions typically assumed to play a role in automatic processes of social cognition. Our results are a step forward in our understanding of the human social brain.

\section{Introduction}

Many animal species display a hierarchical organization of individuals. Perception of cues depicting social hierarchy is biologically determined and it is crucial in regulating survival, reproductive success, health, and social behavior (Cummins, 2000; Boyce, 2004; Sapolsky, 2004). In humans, social hierarchy

Received April 10, 2014; revised May 18, 2015; accepted June 19, 2015.

Author contributions:H.S.-G.,M.B., and N.S.-G. designed research;H.S.-G., M.B., and N.S.-G. performed research; H.S.-G., M.B., and N.S.-G. contributed unpublished reagents/analytic tools; H.S.-G., M.B., and N.S.-G. analyzed data; H.S.-G., M.B., and N.S.-G. wrote the paper.

This work was supported by the Spanish Ministerio de Economía y Competitividad Grant MINEC0-PSI201234071), the European Community's Seventh Framework Programme (Grant FP7/2007-2013)/European Research Council (Grant 323961), and Grup de Recerca en Neurociència Cognitiva, AGAUR (Grant 2014 SGR 1210N). M.B. was funded by a postdoctoral fellowship from the Spanish Ministerio de Economía y Competitividad (MINECO-FPDI2013-17528). N.S.-G. received the prize ICREA Acadèmia for Excellence in Research, funded by the Generalitat de Catalunya.

The authors declare no competing financial interests.

*H.S.-G. and M.B. contributed equally to this work.

Correspondence should be addressed to either Hernando Santamaría-García or Miguel Burgaleta, Center for Brain and Cognition, Department of Technology, Universitat Pompeu Fabra, Roc Boronat 138, 08018, Barcelona, Spain; E-mail: hernando.santamaria@upf.edu or miguel.burgaleta@upf.edu.

DOI:10.1523/JNEUROSCI.1457-14.2015

Copyright $\odot 2015$ the authors $\quad 0270-6474 / 15 / 3510843-08 \$ 15.00 / 0$ refers to the ranking of individuals within a group as a function of power, prestige, status, and resources, and an array of physical features including body postures, facial cues (e.g., eye gaze; Foulsham et al., 2010), sex, age, and attire, among others, can spontaneously convey implicit hierarchical features (Oosterhof and Todorov, 2008; Van Berkum et al., 2008). Nevertheless, knowledge about how the brain perceives such hierarchical cues is limited. A distributed network of brain regions sensitive to social processing has been consistently identified in fMRI studies. This network includes the fusiform cortex, superior temporal gyrus/sulcus, intraparietal sulcus, prefrontal cortex, and the basal ganglia (Grill-Spector et al., 2004; Chiao et al., 2008; Zink et al., 2008). Nevertheless, the temporal dynamics of such structures and the role of their morphology in the perception of social hierarchy remain unexplored.

In the present study, we moved one step forward by focusing on how brain morphology correlates with early markers of hierarchy perception. Understanding how brain structure is related to brain function and behavior is currently of great interest for neuroscience research. Here, we combined eventrelated potentials (ERPs) and structural MRI (sMRI) to assess 
individual differences in fine-grained temporal dynamics of hierarchy perception and to investigate the neuroanatomical correlates of such individual differences. This approach has been used successfully in previous studies in which ERP markers of cognitive abilities were correlated with brain morphology (Walhovd et al., 2005; Fjell et al., 2007; Westlye et al., 2009), although this is the first time that this combination of techniques has been implemented in social neuroscience research.

We built an experimental social hierarchy following a previously validated procedure (Zink et al., 2008; Santamaría-García et al., 2014). Participants performed a visual discrimination task with two covertly simulated players, one with a fixed superior rank (superior player, SP) and the other with a fixed inferior rank (inferior player, IP) with respect to the participant. To measure how participants perceived social hierarchy in others, at each trial, ERPs were recorded while participants saw a photograph of either the SP or IP and an explicit mark depicting hierarchy: three stars for SP and one star for IP (see Materials and Methods). Based on previous studies (SantamaríaGarcía et al., 2014, Chiao et al., 2008), we expected status-related differences (SP vs IP) in ERP components 200 ms, approximately coinciding with the "face-selective" N170 component (Eimer, 2011). However, for the sake of completeness, we also explored potential ERP effects in earlier components observed in previous reports during hierarchy perception in faces such as C1 and P1 (Santamaría-García et al., 2014).

To characterize cortical morphology, we applied surfacebased morphometry and obtained two independent morphometric indices: cortical thickness (CTh) and cortical surface area (CSA). It has been proposed that CTh is sensitive to the number of neuronal bodies, glial and capillary support, and dendritic arborization, whereas CSA may be related to the number and spacing of minicolumnar units of cells (Thompson et al., 2007; la Fougère et al., 2011). Further, subcortical gray matter was investigated by means of subcortical shape analysis (Patenaude et al., 2011). Based on the available fMRI evidence, we hypothesized significant associations between hierarchy-modulated ERP indices and the morphology of brain areas sensitive to early social cues, namely the fusiform cortex, superior temporal gyrus/sulcus, intraparietal sulcus, striatum, and amygdala (Zink et al., 2008; Kumaran et al., 2012).

\section{Materials and Methods \\ Participants}

Twenty-two right-handed participants (11 females) took part in this study. They were undergraduate students recruited at the Universitat Pompeu Fabra (Spain). Mean age was 23.39 ( $\mathrm{SD}=2.4$ years; range $=$ $18-27$ years). Participants were invited through an open call, voluntarily participated, and received 10 Euros per hour. All participants reported normal visual accuracy and none reported psychiatric or neurological conditions. Participants gave their informed consent before inclusion in the study, which conforms to the Code of Ethics of the World Medical Association (Declaration of Helsinki).

\section{Neutral trials}

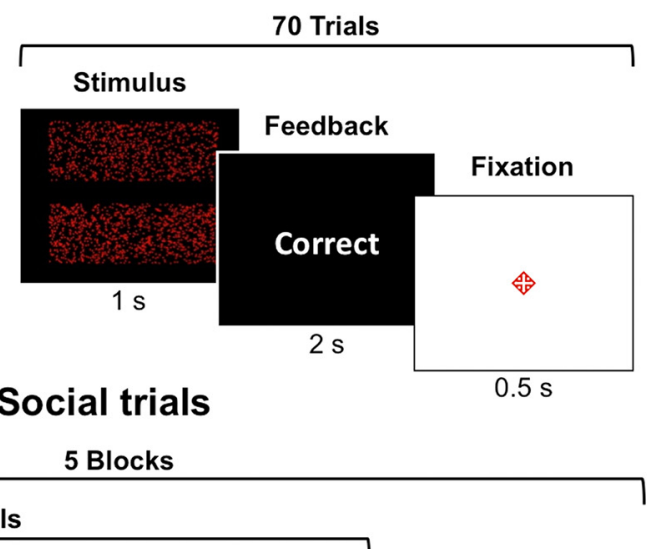

36 Trials

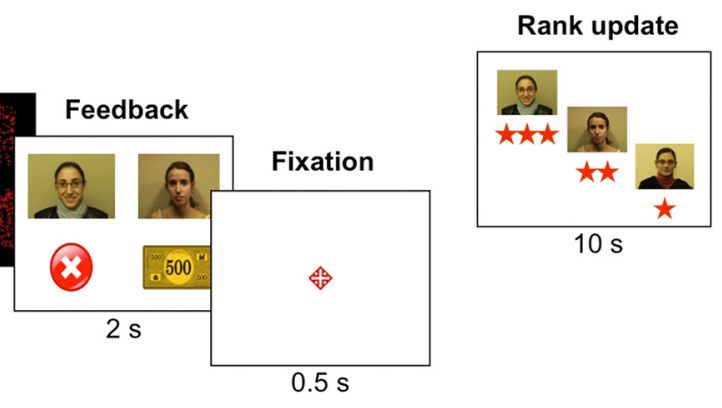

Rank update 
player, was displayed on the screen. Participants had up to $1 \mathrm{~s}$ to decide which rectangle contained more red dots using the corresponding lever (up/down) of a joystick.

Hierarchical stimulus. At the beginning of every trial, we displayed a photograph of the simulated opponent with its corresponding ranking stars (three stars for SP and one star for IP). In the photograph, the simulated player maintained a neutral position and the gaze to the front. In our analyses, we focused on the presentation of these hierarchical stimuli because we considered them to be the most appropriate for the study of social hierarchy perception.

Visual decision task. On a black background, we presented two rectangles of red dots, one at the top of the screen and the other at the bottom. Participants were situated $\sim 50 \mathrm{~cm}$ in front of a 19-inch screen with an angle of vision of $\sim 35^{\circ}$. All dots had the same diameter, shape, and brightness. Each rectangle had a different percentage of red dots, with $>1000$ dots in total. The percentage of red dots was complementary between the rectangles (e.g., if one had $49 \%$ of the dots, the other had $51 \%$; Fig. 1A). In every trial, we displayed screenshots of each rectangle with nine levels of dot percentages $(44,46,48,49,51,52,54$, and 56).

Procedure. To control for possible interactions between sex and hierarchy, male participants played with male-simulated players and female participants with female-simulated players. Participants were first informed that they would play a game based on a visual discrimination task and were then notified that their performance would be compared with that of two players who had already completed the task and that the three players would be ranked according to their performance during the game. Participants were told that their performance could be compared with that of future participants as well.

Participants were situated in an electrically shielded room located in the Neuroscience Laboratory of the Center for Brain and Cognition (Universitat Pompeu Fabra, Barcelona) where EEG activity was registered.

The experiment began after electrode application. First, participants watched a 2 min video of the other players (Fig. 1) to establish the initial hierarchy. Half of the participants were presented with the SP followed by the IP, whereas the other half were presented in the reverse order.

Participants performed 70 practice trials where they solved the numerosity task and received feedback after each response. The game (Fig. 1B) began immediately after training with five blocks of 36 trials ( 180 total, 90 with each simulated player). In each block ( $\sim 5 \mathrm{~min})$, participants played nine consecutive trials twice with each player, followed by an updated ranking presentation. Participants could rest for up to 2 min between blocks. Rank order was fixed by manipulating the SP's or IP's behavior to maintain each participant in the middle of rank. Each trial lasted $\sim 5 \mathrm{~s}$. Each trial started with a $1 \mathrm{~s}$ presentation of the opponent's photograph (hierarchical stimulus) with its corresponding ranking stars. Next, participants performed the visual discrimination task lasting $1 \mathrm{~s}$. Feedback was then presented for $2 \mathrm{~s}$ : pictures of the participant and opponent above, and outcome (a coin meaning correct, an " $\mathrm{X}$ " meaning incorrect or a "time over" message) below. Both players could win or lose in a trial. The trial ended with the fixation cross for $1 \mathrm{~s}$.

\section{EEG/ERP recording}

EEGs were recorded from 31 scalp sites. We placed two bipolar electrodes above and below the participant's left eye to record eye movements, two electrodes on the mastoids, and a reference electrode on the nose. EEG recordings were digitized at $250 \mathrm{~Hz}$. All electrode impedances were $<3$ KOhms. The EEG data were low- and high-pass filtered $(30-0.03 \mathrm{~Hz})$. We have used two different kinds of analyses: stimulus-locked and response-locked analysis. In the stimulus-locked analysis, EEG was segmented into $1100 \mathrm{~ms}$ epochs ranging from $100 \mathrm{~ms}$ before stimulus onset to $1000 \mathrm{~ms}$ after onset (visual discrimination task). Before averaging, segments were baseline corrected by subtracting the mean amplitude of the prestimulus interval $(-100-0 \mathrm{~ms})$. All EEG data were semiautomatically screened offline for eye movements, muscle artifacts, electrode drifting, and amplifier blocking. Segments containing such artifacts were discarded.

\section{Personality and postexperiment questionnaire}

After the experiment and EEG acquisition, participants underwent personality assessment by means of the following standardized scales:
NEO-FI (Costa et al., 1999), BIS-BAS (Carver and White, 1994), and SC-SR (Torrubia et al., 2001). BAS-R and BAS-D scales were combined to measure trait dominance (Carver and White, 1994; Terburg et al., 2011; Hortensius et al., 2014), which is of particular interest because it captures a tendency for assertive, self-assured behaviors and a preference for attaining high status. Furthermore, as a manipulation check (Zink et al., 2008), we asked participants to rate (separately for trials with SP and IP) the task difficulty, how much the other player made the participant motivated, how much the participant liked rounds played with the other player, how important it was for the participant to perform better than the other player, and how influenced participants were by the other player's rank.

\section{MRI acquisition}

Structural MRI was acquired on a $1.5 \mathrm{~T}$ scanner (Signa; GE Medical Systems). A 142-slice, 3D, spoiled gradient-recalled acquisition sequence was obtained in the sagittal plane, with the following acquisition parameters: repetition time $=10.33 \mathrm{~ms}$, echo time $=3.3 \mathrm{~ms}$, inversion time $=$ $600 \mathrm{~ms}$, flip angle $10^{\circ}$, field of view $26 \mathrm{~cm}^{2}$, matrix size $256 \times 256$ pixels, in-plane resolution $0.98 \mathrm{~mm}^{2}$, and slice thickness $1.2 \mathrm{~mm}$ (no gap). Image quality control was performed before image processing.

\section{MRI cortical processing}

MRI images were processed by applying a fully automated pipeline, CIVET 1.1.9 (MacDonald et al., 2000; Kim et al., 2005), for the measurement of regional CT and CSA. CIVET has shown high test-retest reliability (Redolfi et al., 2015), as well as a particularly high geometric/topologic accuracy of cortical anatomy reconstruction compared with other tools (Lee et al., 2006). CIVET was developed at the Montreal Neurological Institute and was applied through the CBRAIN portal (www.cbrain. mcgill.ca). The pipeline comprised several steps, extensively detailed previously (Karama et al., 2009): linear registration of native sMRI to the ICBM152 template (Mazziotta et al., 1995); nonuniformity correction; tissue classification into GM, WM, CSF, and background; pial and white matter surface fitting (40,962 vertices per hemisphere); nonlinear surface registration to a high-resolution surface template in ICBM152 space; inverse registration of the surfaces into native space; CT calculation at each vertex with the $t$-link metric (Lerch and Evans, 2005); CT smoothing applying a $20 \mathrm{~mm}$ FWHM surface-based smoothing kernel; CSA calculation at each vertex as one-third of the total area of all triangular facets adjoining it; and surface area smoothing using a $40 \mathrm{~mm}$ FWHM surface-based smoothing kernel (Lyttelton et al., 2009). Visual quality control of the native cortical surfaces was performed and all surface reconstructions were considered to be successful.

\section{MRI subcortical processing}

We applied the Bayesian Appearance Model (Patenaude et al., 2011), as implemented in FIRST, which is part of the FSL package (FMRIB, Oxford, UK; http://fsl.fmrib.ox.ac.uk/fsl/fslwiki/), to automatically segment the caudate nucleus, the nucleus accumbens, the globus pallidus, the putamen, the amygdala, and the thalamus. FIRST takes into account probabilistic information about the shape and intensity of the segmented structures and has been shown to be highly reliable (Patenaude et al., 2011; Nugent et al., 2013). One advantage of this model over voxel-based morphometry alternatives is that it does not require tissue classification and arbitrary smoothing; instead, it is based directly on the geometry and location of the structure boundary and thus provides a robust estimation of anatomical boundaries and a higher sensitivity to regional variability. Based on manually segmented training data, FIRST creates a surface mesh for each subcortical structure using a deformable mesh model, with the number of vertices for each structure being invariant, thus allowing for vertex-wise comparisons among subjects.

Visual quality control of the subcortical segmentations was performed and one participant was discarded because of poor segmentation of subcortical structures. A total sample size of $N=21$ was included in further steps.

\section{Statistical analysis of ERP and MRI data}

ERP statistical analyses. First, we analyzed the ERP components elicited when participants saw the hierarchical stimulus (photographs and stars 
of SP or IP) by means of an ANOVA with hierarchy (superior and inferior) as within-participant factor and age and sex as nuisance factors. Analyzed ERP signals were averages of different electrodes $(\mathrm{O} 1, \mathrm{O} 2, \mathrm{OZ}$ for $\mathrm{C} 1$ and $\mathrm{P} 1$ components and PO7, PO8, O1, O2, and OZ for N170 component). Second, the ERP components in which we found statistical differences between SP and IP photographs were selected. We then subtracted the component amplitude for SP minus that for IP and used that difference score as an index of the hierarchy effect over that component. The mean averages were computed from at least three electrodes in which the component was located to stabilize the ERP measure.

Statistical analysis of cortical morphology. Cortical vertex-wise analyses were performed via permutation-based inference (Nichols and Holmes, 2002), implemented using the randomize tool of the FSL package of FMRIB (http://fsl.fmrib.ox.ac.uk/fsl/fslwiki/Randomize). For this purpose, the surface data were projected onto an ICBM152 $2 \mathrm{~mm}$ template by means of the appropriate function of the SurfStat Toolbox (http:// www.math.mcgill.ca/keith/surfstat/) so that each vertex value was assigned to its nearest voxel while respecting the standard space of reference. Five thousand permutations were performed for each analysis. The association between difference scores and CT/CSA was analyzed at each vertex of the cortical surface, controlling for the effects of age, sex, and mean CT (for CT analyses) or total CSA (for CSA analyses). Controlling for mean CT or total CSA effects allowed improved sensitivity to detect local areas of significance unrelated to potential overall cortical effects. More specifically, the fitted regression equation at each vertex was as follows:

$$
\mathrm{CT} \sim \mathrm{b}_{0}+\mathrm{b}_{1} \text { Age }+\mathrm{b}_{2} \text { Sex }+\mathrm{b}_{3} \text { MeanCT }+\mathrm{b}_{4} \Delta \mathrm{ERP}+\varepsilon,
$$

where $\triangle \mathrm{ERP}$ represents the difference score for the ERP component of interest. A similar regression equation was fitted for CSA. $t$-statistic maps were produced at each vertex of the cortical surface and the thresholdfree cluster enhancement (TFCE) algorithm was applied to detect cluster-wise statistical signal while avoiding the setting of arbitrary cluster-forming thresholds. Finally, statistical maps were thresholded at $p<0.05$ corrected for multiple comparisons [familywise error (FWE) rate $<5 \%]$.

Statistical analysis of subcortical morphology. Vertex coordinates were analyzed in MNI space, where the structural images were finely registered and structure models were generated. This allowed us to minimize differences in pose and to provide relative deformation indices, orthogonal to the surface, adjusted for total brain size. More specifically, the fitted regression equation at each vertex was as follows:

$$
\mathrm{D}_{\mathrm{T}} \sim \mathrm{b}_{0}+\mathrm{b}_{1} \text { Age }+\mathrm{b}_{2} \text { Sex }+\mathrm{b}_{4} \Delta \mathrm{ERP}+\varepsilon,
$$

where $D_{\mathrm{T}}$ refers to the amount of vertex displacement perpendicular to the fitted surface. $F$ statistics were computed vertex-wise, with the effects of age and sex being partialled out. As was the case for the cortical analyses, vertex-wise analyses were performed via permutation-based inference (Nichols and Holmes, 2002), implemented using the randomize tool of the FSL package of FMRIB. Five thousand permutations were performed for each analysis. The TFCE algorithm was applied to detect cluster-wise statistical signal while avoiding the setting of arbitrary cluster-forming thresholds. Finally, statistical maps were thresholded at $p<0.05$ corrected for multiple comparisons (FWE rate $<5 \%$ ).

\section{Results}

\section{ERP hierarchical modulations during opponent's photograph presentation}

Analysis of the N170 component revealed a main effect of hierarchy $\left(F_{(1,20)}=8.45, p<0.01\right)$. The N170 was larger when participants saw the SP's photograph and stars (Fig. 2). This effect was still significant, although of lower magnitude, when sex and age were included as covariates $\left(F_{(1,21)}=3.31, p<0.05\right)$. Furthermore, we observed a significant sex by hierarchy interaction $\left(F_{(1,21)}=3.96, p<0.05\right)$, with males displaying greater absolute effects compared with females. ANOVAs yielded no significant effects of hierarchy (superior and inferior) over amplitudes of C1 and $\mathrm{P} 1$ components $(p>0.1)$.

To ensure that specific faces were not responsible for the hierarchy effect, we ran an additional ANOVA in which face was also included as a between-participants factor. We found no significant effect of face or face by hierarchy interaction. Because we only found a significant effect over the N170 component, further statistical analyses of MRI data were focused exclusively on $\Delta \mathrm{N} 170$.

To discard the possibility that the observed N170 modulation was due to mere differences in the number of stars displayed, we ran an additional experiment on an independent sample $(n=20$; 16 females; mean age $=21.85, \mathrm{SD}=4.11)$ in which participants were presented with the same faces along with one or three stars, but in this case without a hierarchical context. Here, we hypothesized no significant differences between three- and one-star conditions in N170 amplitude. In this experiment, no profile videos were shown and participants did not perform the visual decision task. Instead, at each trial, a picture was presented, along with 1 or 3 stars, for $1 \mathrm{~s}$. Stimuli were identical to those used in our first experiment. Then, instead of the visual task, a neutral sentence describing the picture was displayed for $2 \mathrm{~s}$ and participants had to respond whether it was true or false. The four possible sentences were "woman and three stars," "woman and one star," "man and three stars," and "man and one star." Half of the presented sentences were true and the other half, false, and were displayed in pseudorandom order. The trial ended with the fixation cross presented for $1 \mathrm{~s}$. The experiment comprised five blocks of 36 trials (180 total, 90 for each "star" condition). Participants could rest for up to $2 \mathrm{~min}$ between blocks. EEG data were recorded and processed as described above (see EEG/ERP recording section) and statistical analyses were performed over the amplitude of the N170 component elicited after the onset of the picture and stars stimuli. We ran an ANOVA with stars ( 3 and 1 ) as the within-participant factor and age and sex as nuisance factors. N170 amplitudes were averaged across PO7, PO8, O1, $\mathrm{O}_{2}$, and OZ. The ANOVA yielded no significant differences in N170 amplitude.

\section{Cortical morphology correlates of hierarchy-modulated ERP components}

We observed a significant positive correlation (FWE-corrected $p<0.05)$ between $\Delta \mathrm{N} 170$ and CT in the right posterior cingulate cortex (MNI coordinates at the peak vertex: $x=-2, y=-20, z=$ 30 ; peak $\beta=0.628, p<0.001$ ). With regard to CSA, a significant positive correlation was found in a region approximately covering the left transverse/superior temporal sulcus/gyrus $(x=64$, $y=-36, z=14$; peak $\beta=0.664, p<0.001$ ), whereas a negative association was observed in a cluster comprising the right insula, ventral anterior temporal cortex, and the anterior part of the fusiform gyrus $(x=-18, y=12, z=-24$; peak $\beta=-0.611$, $p=0.001)$.

\section{Subcortical morphology correlates of hierarchy-modulated ERP components}

We found a significant positive association (FWE-corrected $p<$ 0.05 ) between the magnitude of vertex displacements in the dorsal caudate and $\Delta$ N170 (MNI coordinates at the peak vertex: $x=$ $20, y=-10, z=24)$. Neither negative associations nor other significant relationships were found between $\Delta \mathrm{N} 170$ and the shape of the other analyzed subcortical structures. 

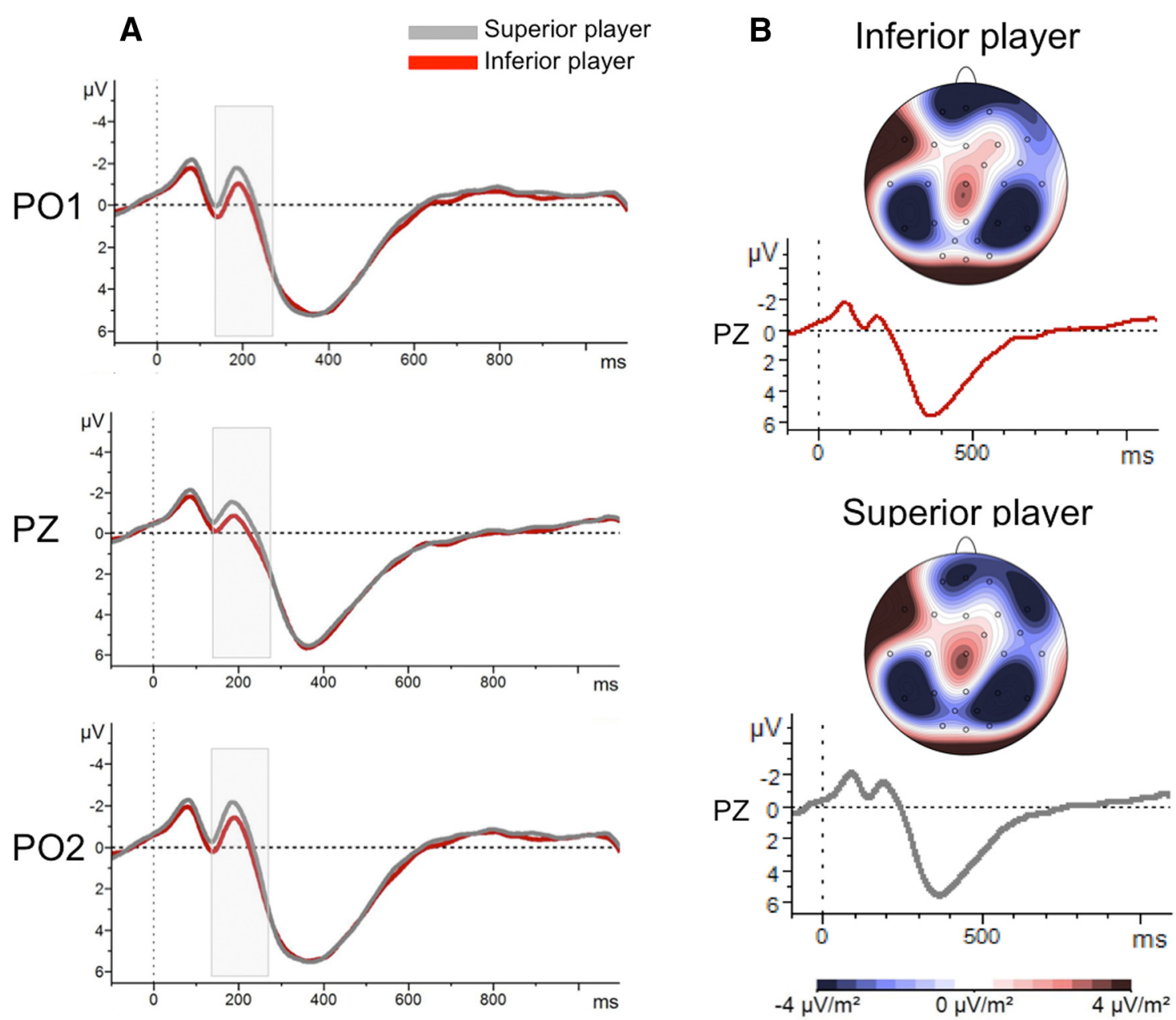

Figure 2. Hierarchical effects in ERP. A, ERP results (photograph stimulus-locked): significant differences took place during the $150-220 \mathrm{~ms}$ window (shaded area), modulating amplitudes of N170 wave. $\boldsymbol{B}$, Pattern of current source density (CSD) for N170 wave (over bilateral parietal occipital areas).

\section{Cortical Thickness}

\section{Cortical Surface Area}
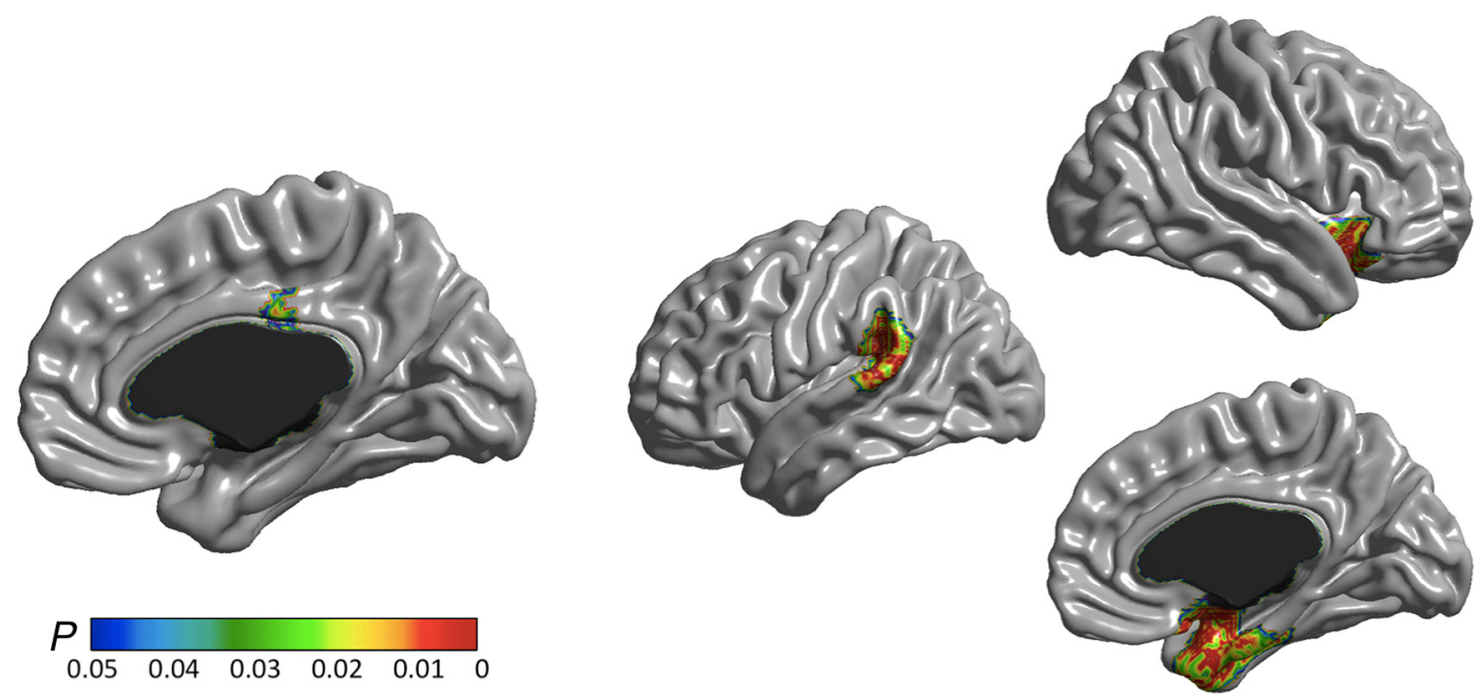

Figure 3. Cortical morphology results. Significant associations between $\Delta \mathrm{N} 170$ and cortical thickness (left) and cortical surface area (right). For CSA, the left picture represents positive associations, whereas right picture depicts negative associations. Results are corrected for multiple comparisons (FWE rate $<5 \%$ ). 


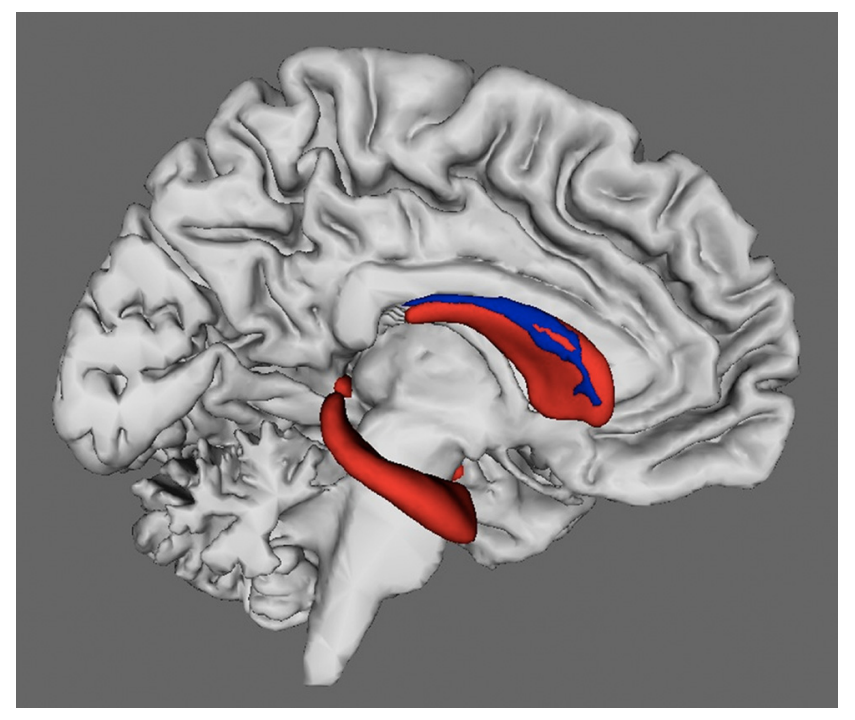

Figure 4. Subcortical morphology results. Significant associations between $\Delta \mathrm{N} 170$ and the shape of the right dorsal caudate nucleus are shown. Results are corrected for multiple comparisons (FWE rate $<5 \%$ ).

\section{Behavioral analyses}

For the sake of completeness, we also evaluated the hierarchy effects on the accuracy and RTs during the visual decision task. We performed $22 \times 2 \times 5$ ANOVAs with sex and age as betweenparticipants factors and hierarchy (superior and inferior) and blocks of game (5) as within-participants factors. The analysis of accuracy did not reveal significant effects or interactions. However, there was a significant main effect of hierarchy over RTs $\left(F_{(1,21)}=9.85, p<0.01\right)$. Participants were faster in trials played in presence of a superior opponent. Interactions between factors did not reach significance. Furthermore, a significant correlation $(r=0.44 p<0.01)$ was observed between the social effect over ERP components $(\Delta \mathrm{N} 170)$ and the social effect over behavioral measures $(\Delta \mathrm{RT})$.

To determine whether the observed experimental effects of social hierarchy were modulated by personality factors, we correlated personality scores from the BIS-BAS, NEO-FI, and SC-SR scales with $\Delta \mathrm{N} 170$ and $\Delta \mathrm{RT}$. $\Delta \mathrm{N} 170$ was significantly correlated with trait dominance scores $(r=0.39, p<0.05)$. At the behavioral level, a significant correlation was also detected between $\Delta \mathrm{RT}$ and trait dominance $(r=0.36, p<0.05)$. No significant correlations were observed between experimental effects and the other personality measures.

Regarding our postexperiment questionnaire, we observed that participants found that playing with SP was significantly more motivating $\left(t_{(17)}=5.66, p<0.001\right)$ and enjoyable $\left(t_{(17)}=12.85, p<\right.$ $0.001)$ than with IP, felt more influenced by SP's rank $\left(t_{(17)}=7.16\right.$, $p<0.001$ ), and considered it more important to outperform SP than IP $\left(t_{(17)}=17.61, p<0.001\right)$. However, participants did not perceive differences in task difficulty depending on the opponent's rank $\left(t_{(17)}=1.14, p=0.120\right)$. (Note that this information was not available for four participants due to data loss.)

\section{Discussion}

In the present study, we combined ERPs and sMRI to reveal for the first time the cortical and subcortical anatomical substrates of early social status recognition. This combination of techniques provided a unique approach for understanding the neuroanatomical basis of social hierarchy processing in humans because it allowed us to isolate a fine-grained ERP-based measure of sensi- tivity to a hierarchical context that could be regressed against structural indices of brain morphology.

In a first stage, we observed the expected modulations of social hierarchy over the N170 component, so that higher amplitudes were observed when we presented a picture of a SP along with his or her corresponding status mark (three stars) than when we presented a picture of an IP accompanied by one star. We thus replicated Chiao et al. (2008), who demonstrated that humans are able to extract dominance and submission information from faces as early as $200 \mathrm{~ms}$. Furthermore, our results fit well with previous reports showing that N170 is not only involved in the process of face recognition (Vuilleumier and Pourtois, 2007; Eimer, 2011), but has also been linked to the identification of symbolic features such as social information in faces (Eimer, 2011; Meaux et al., 2014).

In a second stage, we focused on the association between brain morphology and social hierarchy modulations of N170. $\Delta \mathrm{N} 170$ correlated, at the cortical level, with the morphology of the superior temporal sulcus (STS), the right posterior cingulate cortex, and a cluster of areas comprising the anteroventral temporal lobe, fusiform gyrus, and right insula and, at the subcortical level, with the morphology of the caudate nucleus.

Concerning the cortical correlates, previous studies have shown that STS is sensitive to social and emotional cues in faces (Marsh et al., 2009; Muscatell et al., 2012; Mende-Siedlecki et al., 2013; Stanley and Adolphs, 2013), whereas the fusiform gyrus includes the so-called fusiform face area (lateral part of right fusiform gyrus), which in the social domain is known for being involved in the recognition of individual identity (Kanwisher and Yovel, 2006; Kawasaki et al., 2012). Furthermore, posterior cingulate cortex and insula are relevant for recognizing contextual social information associated to faces (Eisenberger et al., 2003; Muscatell et al., 2012), as well as for face categorization according to their learned social status (Eger et al., 2013). Interestingly, the reported correlations in the temporal-fusiform-insular cluster between $\Delta \mathrm{N} 170$ and brain morphology were negative, which could be interpreted in light of the possibility that a reduced morphometric index (in this case, CSA) may be linked to a decreased functional activity (Zatorre et al., 2012). In this vein, low insular activity has been linked with reduced inequity aversion (Hsu et al., 2008), as well as with a preference for social distance judgments (e.g., hierarchical relations; Farrow et al., 2011). These negative correlations may also reflect a higher sensitivity to an emotional component likely involved in the hierarchy perception. Indeed, hierarchy recognition is associated with increased arousal, nervousness, high stress, and emotional reactivity (Boyce, 2004; Mehta et al., 2008), and previous fMRI studies showed that negative affectivity increases with deactivation of a network comprising the temporal pole, right insula, and fusiform gyrus, among other areas (Pérez-Edgar et al., 2007; Kret et al., 2011). Therefore, it is possible that a reduced insular region entails an increased preference and/or reactivity to hierarchical settings.

At the subcortical level, we found a positive correlation between $\Delta \mathrm{N} 170$ and the right caudate nucleus. This result is consistent with the available fMRI evidence showing that the caudate is involved in the perception of a social hierarchy (Britton et al., 2006; Zink et al., 2008). Previous studies have shown that the evaluation of hierarchical identity in faces is a highly salient process (Zink et al., 2008; Menon and Uddin, 2010) that depends in part on the recruitment of structures implicated in reward processing (Ly et al., 2011). Along this line, the caudate has been suggested to play a role in detecting salient socio-emotional in- 
formation in faces (Zink et al., 2008; Menon and Uddin, 2010), as well as in spotting predictors of rewards (Zink et al., 2008; Menon and Uddin, 2010; Ly et al., 2011). Therefore, variability in the morphology of the right caudate may be related to the sensitivity to detect salient hierarchical cues that are potentially rewarding.

Some considerations must be made with regard to the differences between cortical thickness and surface area given that $\mathrm{CT}$ was related to $\Delta \mathrm{N} 170$ in the cingulate cortex, whereas CSA covaried with $\Delta \mathrm{N} 170$ in the STS, insular cortex, and fusiform gyrus. CT is generally considered as an index of neuron density, dendritic arborization, and glial support; conversely, CSA is thought to depend on the number and spacing of neuronal minicolumns (Thompson et al., 2007; la Fougère et al., 2011). A straightforward consequence of such distinction is that the reported results may differ in the microstructural features that drive the observed anatomical correlations. An important implication of this possible difference in cellular underpinnings is that some of the associations found are more prone to be explained by environmental effects, whereas others would be driven by genetic factors to a higher extent. More specifically, CT associations may be more sensitive to environmental pressures, given that CT-related microstructures such as glial and capillary support, as well as dendritic arborization (Thompson et al., 2007), dendritic and spine rearrangement and elimination (Petanjek et al., 2008), and gliogenesis (Dong and Greenough, 2004) are sensitive to learning and experience, whereas CSA-related results are likely to be under a higher genetic control, given that CSA is thought to depend on neurogenesis and neuronal migration and such processes are known to suffer little modifications after term gestation (Hill et al., 2010). Because most of the results at the cortical level of analysis were found for CSA, the question arises of to what extent is social status recognition under genetic control and whether appropriate training would allow modulating the effect of social hierarchy over other cognitive processes (Santamaría-García et al., 2014).

It is noteworthy that we did not observe our hypothesized association between amygdalar morphology and hierarchy effects over N170. As Utevsky and Platt (2014) pointed out, it remains unclear which aspects of social hierarchy perception are under amygdalar influence, which may affect a variety of processes, including the representation of one's own status within a social hierarchy (Zink et al., 2008; Kumaran et al., 2012), behaviors that play an indirect role in social processes such as gaze following (Kawashima et al., 1999) and theory of mind (Baron-Cohen et al., 1999) or individual differences in related personality traits, such as fear or agression (Kling and Cornell, 1971; Antoniadis et al., 2007). Therefore, it is not surprising that amygdalar morphology is not directly involved in the specific fast-paced perception of social hierarchies studied here.

An interesting observation that should be studied in future research is that the pool of corticosubcortical areas identified here has been described previously as involved in the automatic, rather than controlled, integration of social information (Lieberman, 2007; Yang and Li, 2012). Automatic processes are defined as early, fast, and unaware and are related to activity in a dorsal network that includes, but is not limited to, all of the regions for which we observed an association between morphology and $\Delta \mathrm{N} 170$. In contrast, controlled processes are considered as intentional, susceptible to interruption, and related to a frontal network comprising the prefrontal cortex, orbitofrontal cortex, and anterior cingulate cortex (Lieberman, 2007; Yang and Li, 2012). Therefore, future studies should address empirically the extent to which $\Delta \mathrm{N} 170$ is indexing automatic, rather than controlled, recognition of a hierarchical structure or status.

\section{Conclusion}

In the present study, we reveal for the first time the cortical and subcortical anatomical substrates of early social status recognition. We conclude that such early processes are captured by the N170 component, likely reflecting the first stages in the inference of social identity, and that individual differences in the hierarchical effect over N170 is predicted by the morphology of a corticosubcortical network previously related to social cognition.

\section{References}

Antoniadis EA, Winslow JT, Davis M, Amaral DG (2007) Role of the primate amygdala in fear potentiated startle: effects of chronic lesions in the rhesus monkey. J Neurosci 27:7386-7396. CrossRef Medline

Baron-Cohen S, Ring HA, Wheelwright S, Bullmore ET, Brammer MJ, Simmons A, Williams SC (1999) Social intelligence in the normal and autistic brain: an fMRI study. Eur J Neurosci 11:1891-1898. CrossRef Medline

Boyce WT (2004) Social stratification, health, and violence in the very young. Ann N Y Acad Sci 1036:47-68. Medline

Britton JC, Phan KL, Taylor SF, Welsh RC, Berridge KC, Liberzon I (2006) Neural correlates of social and nonsocial emotions: an fMRI study. Neuroimage 31:397-409. CrossRef Medline

Carver CS, White TL (1994) Behavioral inhibition, behavioral activation, and affective responses to impending reward and punishment: the BIS/ BAS scales. Journal of Personality and Social Psychology 67:319-333. CrossRef

Chiao JY, Adams RB, Tse PU, Lowenthal L, Richeson JA, Ambady N (2008) Knowing who's boss: $\mathrm{AMRI}$ and ERP investigations of social dominance perception. Group Process Intergroup Relat 11:201-214. CrossRef Medline

Costa Jr PT, McCrae RR (1992) NEO personality inventory-revised (NEO PI-r) and NEO five-factor inventory (NEO-FFI) professional manual. Odessa, FL: Psychological Assessment Resources.

Cummins D (2000) How the social envoironment shaped the evolution of the mind. Synthese, 122 (1/2), 3-28.

Dong WK, Greenough WT (2004) Plasticity of nonneuronal brain tissue: roles in developmental disorders. Ment Retard Dev Disabil Res Rev 10: 85-90. CrossRef Medline

Eger E, Moretti L, Dehaene S, Sirigu A (2013) Decoding the representation of learned social roles in the human brain. Cortex 49:2484-2493. CrossRef Medline

Eimer M (2011) The face-sensitivity of the N170 component. Front Hum Neurosci 5:119. Medline

Eisenberger NI, Lieberman MD, Williams KD (2003) Does rejection hurt? An FMRI study of social exclusion. Science 302:290-292. CrossRef Medline

Farrow TF, Jones SC, Kaylor-Hughes CJ, Wilkinson ID, Woodruff PW, Hunter MD, Spence SA (2011) Higher or lower? The functional anatomy of perceived allocentric social hierarchies. Neuroimage 57:15521560. CrossRef Medline

Fjell AM, Walhovd KB, Fischl B, Reinvang I (2007) Cognitive function, P3a/ P3b brain potentials, and cortical thickness in aging. Hum Brain Mapp 28:1098-1116. CrossRef Medline

Foulsham T, Cheng JT, Tracy JL, Henrich J, Kingstone A (2010) Gaze allocation in a dynamic situation: effects of social status and speaking. Cognition 117:319-331. CrossRef Medline

Grill-Spector K, Knouf N, Kanwisher N (2004) The fusiform face area subserves face perception, not generic within-category identification. Nat Neurosci 7:555-562. CrossRef Medline

Hill J, Inder T, Neil J, Dierker D, Harwell J, Van Essen D (2010) Similar patterns of cortical expansion during human development and evolution. Proc Natl Acad Sci U S A 107:13135-13140. CrossRef Medline

Hortensius R, van Honk J, de Gelder B, Terburg D (2014) Trait dominance promotes reflexive staring at masked angry body postures. PLoS One 9:e116232. CrossRef Medline

Hsu M, Anen C, Quartz SR (2008) The right and the good: distributive justice and neural encoding of equity and efficiency. Science 320:10921095. CrossRef Medline

Kanwisher N, Yovel G (2006) The fusiform face area: a cortical region specialized for the perception of faces. Philos Trans R Soc Lond B Biol Sci 361:2109-2128. CrossRef Medline

Karama S, Ad-Dab’bagh Y, Haier RJ, Deary IJ, Lyttelton OC, Lepage C, Evans AC; Brain Development Cooperative Group (2009) Positive association between 
cognitive ability and cortical thickness in a representative US sample of healthy 6 to 18 year-olds. Intelligence 37:145-155. CrossRef Medline

Kawasaki H, Tsuchiya N, Kovach CK, Nourski KV, Oya H, Howard MA, Adolphs R (2012) Processing of facial emotion in the human fusiform gyrus. J Cogn Neurosci 24:1358-1370. CrossRef Medline

Kawashima R, Sugiura M, Kato T, Nakamura A, Hatano K, Ito K, Fukuda H, Kojima S, Nakamura K (1999) The human amygdala plays an important role in gaze monitoring: a PET study. Brain 122:779-783. CrossRef Medline

Kim JS, Singh V, Lee JK, Lerch J, Ad-Dab'bagh Y, MacDonald D, Lee JM, Kim SI, Evans AC (2005) Automated 3-D extraction and evaluation of the inner and outer cortical surfaces using a Laplacian map and partial volume effect classification. Neuroimage 27:210-221. CrossRef Medline

Kling A, Cornell R (1971) Amygdalectomy and social behavior in the caged stump-tailed macaque (Macaca speciosa). Folia Primatol (Basel) 14:190208. CrossRef Medline

Kret ME, Denollet J, Grèzes J, de Gelder B (2011) The role of negative affectivity and social inhibition in perceiving social threat: an fMRI study. Neuropsychologia 49:1187-1193. CrossRef Medline

Kumaran D, Melo HL, Duzel E (2012) The emergence and representation of knowledge about social and nonsocial hierarchies. Neuron 76:653-666. CrossRef Medline

la Fougère C, Grant S, Kostikov A, Schirrmacher R, Gravel P, Schipper HM, Reader A, Evans A, Thiel A (2011) Where in-vivo imaging meets cytoarchitectonics: the relationship between cortical thickness and neuronal density measured with high-resolution [18F]flumazenil-PET. Neuroimage 56:951-960. CrossRef Medline

Lee JK, Lee JM, Kim JS, Kim IY, Evans AC, Kim SI (2006) A novel quantitative cross-validation of different cortical surface reconstruction algorithms using MRI phantom. Neuroimage 31:572-584. CrossRef Medline

Lerch JP, Evans AC (2005) Cortical thickness analysis examined through power analysis and a population simulation. Neuroimage 24:163-173. CrossRef Medline

Lieberman MD (2007) Social cognitive neuroscience: a review of core processes. Annu Rev Psychol 58:259-289. CrossRef Medline

Ly M, Haynes MR, Barter JW, Weinberger DR, Zink CF (2011) Subjective socioeconomic status predicts human ventral striatal responses to social status information. Curr Biol 21:794-797. CrossRef Medline

Lyttelton OC, Karama S, Ad-Dab'bagh Y, Zatorre RJ, Carbonell F, Worsley K, Evans AC (2009) Positional and surface area asymmetry of the human cerebral cortex. Neuroimage 46:895-903. CrossRef Medline

MacDonald D, Kabani N, Avis D, Evans AC (2000) Automated 3-D extraction of inner and outer surfaces of cerebral cortex from MRI. Neuroimage 12:340-356. CrossRef Medline

Marsh AA, Blair KS, Jones MM, Soliman N, Blair RJ (2009) Dominance and submission: the ventrolateral prefrontal cortex and responses to status cues. J Cogn Neurosci 21:713-724. CrossRef Medline

Mazziotta JC, Toga AW, Evans A, Fox P, Lancaster J (1995) A probabilistic atlas of the human brain: theory and rationale for its development: the International Consortium for Brain Mapping (ICBM). Neuroimage 2:89-101. CrossRef Medline

Meaux E, Roux S, Batty M (2014) Early visual ERPs are influenced by individual emotional skills. Soc Cogn Affect Neurosci 9:1089-1098. Medline

Mehta PH, Jones AC, Josephs RA (2008) The social endocrinology of dominance: basal testosterone predicts cortisol changes and behavior following victory and defeat. J Pers Soc Psychol 94:1078-1093. CrossRef Medline

Mende-Siedlecki P, Said CP, Todorov A (2013) The social evaluation of faces: a meta-analysis of functional neuroimaging studies. Soc Cogn Affect Neurosci 8:285-299. CrossRef Medline

Menon V, Uddin LQ (2010) Saliency, switching, attention and control: a network model of insula function. Brain Struct Funct 214:655-667. CrossRef Medline

Muscatell KA, Morelli SA, Falk EB, Way BM, Pfeifer JH, Galinsky AD, Lieberman MD, Dapretto M, Eisenberger NI (2012) Social status modulates neural activity in the mentalizing network. Neuroimage 60:1771-1777. CrossRef Medline

Nichols TE, Holmes AP (2002) Nonparametric permutation tests for func- tional neuroimaging: a primer with examples. Hum Brain Mapp 15:1-25. CrossRef Medline

Nugent AC, Luckenbaugh DA, Wood SE, Bogers W, Zarate CA Jr, Drevets WC (2013) Automated subcortical segmentation using FIRST: Testretest reliability, interscanner reliability, and comparison to manual segmentation. Hum Brain Mapp 34:2313-2329. CrossRef Medline

Oosterhof NN, Todorov A (2008) The functional basis of face evaluation. Proc Natl Acad Sci U S A 105:11087-11092. CrossRef Medline

Patenaude B, Smith SM, Kennedy DN, Jenkinson M (2011) A Bayesian model of shape and appearance for subcortical brain segmentation. Neuroimage 56:907-922. CrossRef Medline

Pérez-Edgar K, Roberson-Nay R, Hardin MG, Poeth K, Guyer AE, Nelson EE, McClure EB, Henderson HA, Fox NA, Pine DS, Ernst M (2007) Attention alters neural responses to evocative faces in behaviorally inhibited adolescents. Neuroimage 35:1538-1546. CrossRef Medline

Petanjek Z, Judas M, Kostović I, Uylings HB (2008) Lifespan alterations of basal dendritic trees of pyramidal neurons in the human prefrontal cortex: a layer-specific pattern. Cereb Cortex 18:915-929. CrossRef Medline

Redolfi A, Manset D, Barkhof F, Wahlund LO, Glatard T, Mangin JF, Frisoni GB; neuGRID Consortium, for the Alzheimer's Disease Neuroimaging Initiative (2015) Head-to-head comparison of two popular cortical thickness extraction algorithms: a cross-sectional and longitudinal study. PLoS One 10:e0117692. CrossRef Medline

Santamaría-García H, Pannunzi M, Ayneto A, Deco G, Sebastián-Gallés N (2014) "If you are good, I get better": the role of social hierarchy in perceptual decisionmaking. Soc Cogn Affect Neurosci 9:1489-1497. Medline

Sapolsky RM (2004) Social status and health in humans and other animals. Annu Rev Anthropol 33:393-418. CrossRef

Stanley DA, Adolphs R (2013) Toward a neural basis for social behavior. Neuron 80:816-826. CrossRef Medline

Terburg D, Hooiveld N, Aarts H, Kenemans JL, van Honk J (2011) Eye tracking unconscious face-to-face confrontations: dominance motives prolong gaze to masked angry faces. Psychol Sci 22:314-319. CrossRef Medline

Thompson PM, Hayashi KM, Dutton RA, Chiang MC, Leow AD, Sowell ER, De Zubicaray G, Becker JT, Lopez OL, Aizenstein HJ, Toga AW (2007) Tracking Alzheimer's disease. Ann N Y Acad Sci 1097:183-214. CrossRef Medline

Torrubia R, Avila C, Moltó J y Caseras X (2001) The sensitivity to punishment and sensitivity reward questionnaire (SPSRQ) as a measure of Gray's anxiety and impulsivity dimensions. Personality and Individual Differences 31 837-862.

Utevsky AV, Platt ML (2014) Status and the brain. PLoS Biol 12:e1001941. CrossRef Medline

Van Berkum JJ, van den Brink D, Tesink CM, Kos M, Hagoort P (2008) The neural integration of speaker and message. J Cogn Neurosci 20:580-591. CrossRef Medline

Vuilleumier P, Pourtois G (2007) Distributed and interactive brain mechanisms during emotion face perception: evidence from functional neuroimaging. Neuropsychologia 45:174-194. CrossRef Medline

Walhovd KB, Fjell AM, Reinvang I, Lundervold A, Fischl B, Salat D, Quinn BT, Makris N, Dale AM (2005) Cortical volume and speed-ofprocessing are complementary in prediction of performance intelligence. Neuropsychologia 43:704-713. CrossRef Medline

Westlye LT, Walhovd KB, Bjornerud A, Due-Tønnessen P, Fjell AM (2009) Errorrelated negativity is mediated by fractional anisotropy in the posterior cingulate gyrus-a study combining diffusion tensor imaging and electrophysiology in healthy adults. Cereb Cortex 19:293-304. CrossRef Medline

Yang J, Li P (2012) Brain networks of explicit and implicit learning. PLoS One 7:e42993. CrossRef Medline

Zatorre RJ, Fields RD, Johansen-Berg H (2012) Plasticity in gray and white: neuroimaging changes in brain structure during learning. Nat Neurosci 15:528-536. CrossRef Medline

Zink CF, Tong Y, Chen Q, Bassett DS, Stein JL, Meyer-Lindenberg A (2008) Know your place: neural processing of social hierarchy in humans. Neuron 58:273-283. CrossRef Medline 